\title{
Male Gender Is Associated with Lymph Node Metastasis but Not with Recurrence in Papillary Thyroid Carcinoma
}

\author{
Jiang Zhu $\mathbb{D}^{1,2}$ Rui Huang $\mathbb{D}^{3}{ }^{3}$ Ping Yu, ${ }^{1}$ Haoyu Ren $\mathbb{D}^{1},{ }^{1}$ and Xinliang Su $\mathbb{D}^{1}$ \\ ${ }^{1}$ Department of Endocrine and Breast Surgery, The First Affiliated Hospital of Chongqing Medical University, Chongqing, China \\ ${ }^{2}$ Department of Liver Surgery, Liver Transplantation Division, West China Hospital, Sichuan University, Chengdu, China \\ ${ }^{3}$ Department of Anesthesiology, The Second Affiliated Hospital, Zhejiang University School of Medicine, Hangzhou, China
}

Correspondence should be addressed to Xinliang Su; suxinliang@21cn.com

Received 3 December 2021; Accepted 1 February 2022; Published 28 February 2022

Academic Editor: Claudio Casella

Copyright (c) 2022 Jiang Zhu et al. This is an open access article distributed under the Creative Commons Attribution License, which permits unrestricted use, distribution, and reproduction in any medium, provided the original work is properly cited.

Background. The incidence of papillary thyroid carcinoma (PTC) is higher in females than in males, but it remains unclear whether gender is associated with the aggressiveness of this disease. We aimed to clarify the influence of gender on the risk of developing lymph node metastasis (LNM) and on the prognosis of PTC patients. Study Design. Retrospective cohort study. Setting. Academic tertiary care center. Methods. Clinical data of PTC patients who were admitted to the Department of Endocrine and Breast Surgery, the First Affiliated Hospital of Chongqing Medical University, between January 2013 and December 2018 were retrospectively reviewed. The differences in clinical features and outcomes between female and male patients were compared. Univariate and multivariate logistic regression analyses were conducted to assess the impact of gender on LNM. Kaplan-Meier curves were used to estimate recurrence-free survival (RFS). Results. A total of consecutive 2536 patients were enrolled in this study. Males accounted for $25.2 \%$ (639 cases) of all patients. Central lymph node metastasis (CLNM) and lateral lymph node metastasis (LLNM) rates were 52.5\% (1346/2536) and 22.0\% (558/2536), respectively. Male presented with higher LNM rates than female patients $(65.7 \%$ vs. $51.2 \% ; P<0.001)$. Male gender was independently associated with LNM (OR $=1.93,95 \%$ CI: $1.59-2.35$; $P<0.001)$. After full adjustment, male gender still remained significantly associated with CLNM in all subgroups; however, subgroup analyses indicated no significant relationship between gender and LLNM. In addition, after a median follow-up period of 30 months, no significant difference was found in RFS between female and male patients $(P=0.15)$. Conclusions. This observational cohort study revealed that male gender was significantly associated with CLNM; whereas, LLNM was not different between female and male PTC patients in southwestern China. Moreover, currently, there is insufficient evidence to justify that male gender is an independent prognostic factor for recurrence.

\section{Introduction}

The incidence of papillary thyroid carcinoma (PTC) has increased rapidly in recent years [1-3]. Similar to some other tumors [4], a differing incidence of thyroid cancer between genders is apparent. It is well-known that there are approximately three times as many females suffering from thyroid cancer as males [5-7]. However, male gender is reported to be a poor prognostic factor in PTC, with notably higher death and recurrence rates [8-12]. On the contrary, some prior studies suggested that male gender is not associated with tumor aggressiveness or lymph node status [13-15]. Therefore, it is not easy to make an appropriate therapeutic decision according to these contradictory results.

In fact, PTC is a heterogeneous disease $[7,16]$, and the risk of lymph node metastasis (LNM) varies with the differing clinicopathological features of each patient [17-19]. LNM in PTC patients is common, which may lead to tumor relapse and repeat surgery $[20,21]$. To date, the relationship of gender with clinicopathological characteristics and prognosis in PTC patients has not been well defined, particularly regarding LNM, and reports on this issue are limited.

Thus, we aimed to evaluate the impact of gender on LNM and assess the risk of recurrence in subgroups of PTC with 
various clinicopathological characteristics based on realworld data of thyroid cancer cases from southwest China.

\section{Methods}

2.1. Patients. Medical records of patients between January 2013 and December 2018 at the Department of Endocrine and Breast Surgery, the First Affiliated Hospital of Chongqing Medical University, were reviewed and collected. The inclusion criteria were as follows: (a) age 18-80y, (b) initial thyroid surgery, and (c) histologically diagnosed with PTC. Patients with incomplete clinical data or distant metastasis were excluded from this study.

2.2. Treatment and Follow-Up. As described previously, our PTC patients underwent standard treatment procedures: lobectomy plus ipsilateral central compartment lymph node dissection which is recommended by Chinese guideline [22-24]. In our center, intraoperative frozen section of ipsilateral central lymph nodes was routinely used. Total thyroidectomy plus ipsilateral central compartment lymph node dissection was indicated for PTC patients with LNM, extrathyroid extension (ETE), bilaterality, or tumor size $>20 \mathrm{~mm}$. The decision to perform lateral lymph node dissection was comprehensive, determined based on preoperative ultrasound-reported LN status, tumor size and location, ETE, and intraoperative frozen section results. Radioactive iodine therapy and thyroid stimulating hormone suppression were recommended for individuals when clinically indicated.

Our preoperative ultrasound report consisted of the following clinicopathological parameters: the location and size of tumor, multifocality, bilaterality, chronic lymphocytic thyroiditis (CLT), and clinical $\mathrm{N}(\mathrm{cN})$ stage: $\mathrm{cN} 0, \mathrm{cN} 1 \mathrm{a}$, and $\mathrm{cN} 1 \mathrm{~b}$. The details of intraoperative findings and surgery procedures were carefully recorded by surgeons. All surgical specimens were sent for postoperative histopathology. $\mathrm{Pa}-$ thology reports included tumor size, multifocality, ETE, chronic lymphocytic thyroiditis (CLT), number of central lymph node metastasis (CLNM), and lateral lymph node metastasis (LLNM).

Postoperative patients regularly came back to the outpatient for a check every 3 months for 1 year after initial therapy, followed by one visit every 6 months. The follow-up examination items included serum levels of triiodothyronine (T3), thyroxine (T4), thyrotrophin (TSH), thyroglobulin $(\mathrm{Tg})$, thyroid globulin antibody (TgAb), and neck ultrasound. The definition of recurrent disease was a nonstimulated $\mathrm{Tg}$ level of $\geq 0.2 \mathrm{ng} / \mathrm{mL}$, stimulated $\mathrm{Tg}>1 \mathrm{ng} / \mathrm{mL}$, or evidence of disease by neck imaging during follow-up time [12]. Recurrence-free survival (RFS) was defined as the time from the initial surgery to the discovery of cancer recurrence or the most recent follow-up.

2.3. Statistical Analysis. Categorical and continuous variables between male and female patients were analyzed using Fisher's exact test and Student's $t$-test, respectively. Multivariable logistic regression analyses with a backward stepdown selection were performed to identify the independent risk factors of lymph node metastasis. We also conducted stratified analyses to assess the relationship between gender and CLNM or LLNM. Kaplan-Meier curves and the log-rank test were used to calculate RFS. Data analyses were performed using $R$ software (v. 3.4.3). All statistical tests were considered significant for $P<0.05$.

\section{Results}

3.1. Patient Clinical Pathological Characteristics. Overall, a total of 2536 cases were included in this study. The average age of the total study population was $42.9 \pm 11.9 \mathrm{y}$ (mean \pm SD). Additionally, 639 (25.2\%) were males and 1897 (74.8\%) were females. Demographic and clinicopathologic characteristics of patients with PTC are given in Table 1. Papillary thyroid microcarcinoma (PTMC) patients accounted for $56.3 \%$ of all patients. ETE and CLT were detected in 865 (34.1\%) and 526 (20.7\%) patients, respectively. LNM occurred in $54.9 \%$ of patients. CLNM and LLNM rates were 52.5\% (1346/2536) and 22.0\% (558/2536), respectively. Recurrence occurred in 29 patients (1.1\%).

The female group had higher percentages of CLT $(10.6 \%$ vs. $24.1 \% ; P<0.001)$ than the male group, while CLNM (64.2\% vs. $48.6 \% ; P<0.001)$ and LLNM $(26.3 \%$ vs. $20.6 \%$; $P<0.001)$ occurred more frequently in the male group. No differences were found in age, tumor size, ETE, multifocality, and recurrence between male and female groups (all $P>0.05)$.

3.2. Univariate and Multivariate Logistic Regression Analyses of PTC Patients. Associations between LNM and the potential risk factors are given in Table 2. Univariate analysis showed that male gender, age, tumor size, ETE, multifocality, and bilaterality were significantly associated with LNM. However, tumor location and CLT showed no significant association with LNM. In multivariate logistic regression analysis, male gender $(\mathrm{OR}=1.93,95 \% \mathrm{CI}$ : $1.59-2.35 ; \quad P<0.001)$, larger tumor size $(10-20 \mathrm{~mm}$, $\mathrm{OR}=2.09, \quad 95 \% \quad \mathrm{CI}: \quad 1.75-2.52 ; \quad P<0.001) \quad(>20 \mathrm{~mm}$, $\mathrm{OR}=4.18,95 \% \mathrm{CI}: 3.06-5.73 ; P<0.001), \mathrm{ETE}(+)(\mathrm{OR}=1.31$, 95\% CI: $1.10-1.57 ; \quad P=0.003)$, and multifocality(+) (OR $=1.92$, 95\% CI: 1.53-2.41; $P<0.001)$ were independent risk factors of LNM. On the contrary, when compared to age $<35 \mathrm{y}$, being age $>55 \mathrm{y}(\mathrm{OR}=0.42,95 \% \mathrm{CI}: 0.33-0.55$, $P<0.001)$ or age $35-55$ y $(\mathrm{OR}=0.58,95 \% \mathrm{CI}: 0.48-0.70$; $P<0.001)$ were protective factors for LNM.

Comparisons of LNM rates between male and female patients stratified according to age and tumor size group are shown in Figure 1. Clearly, the overall rate of LNM in younger male patients was significantly greatest in all groups. Similar differences were also observed in patients aged $35-55 \mathrm{y}(62.1 \%$ vs. $48.1 \% ; P<0.001)$ and patients aged $>55$ y: $(63.5 \%$ vs. $41.8 \%$; $P<0.001)$. Furthermore, general trends indicated that lymph node positive rates increased with larger tumors, with male subgroups of tumor size $>20 \mathrm{~mm}$ having the highest LNM rate of $92.2 \%$. The proportion of positive lymph nodes ranged from $56.7 \%$ among 
TABle 1: Demographic and clinicopathologic characteristics of male and female papillary thyroid carcinoma patients.

\begin{tabular}{|c|c|c|c|c|}
\hline Characteristics & Total $(N=2536)$, no. $(\%)$ & Male $(N=639)$ & Female $(N=1897)$ & $P$ value \\
\hline Age (y) & $42.9 \pm 11.9$ & $42.7 \pm 11.4$ & $42.9 \pm 12.2$ & 0.586 \\
\hline$<35$ & $712(28.1)$ & $176(27.5)$ & $536(28.3)$ & 0.607 \\
\hline $35-55$ & $1417(55.9)$ & $367(57.5)$ & $1050(55.3)$ & \\
\hline$\geq 55$ & $407(16.0)$ & $96(15.0)$ & $311(16.4)$ & \\
\hline Tumor size (mm) & $12.1 \pm 8.3$ & $11.9 \pm 7.9$ & $12.2 \pm 8.4$ & 0.612 \\
\hline$\leq 10$ & $1429(56.3)$ & $353(55.2)$ & $1076(56.7)$ & 0.397 \\
\hline $10-20$ & $830(32.7)$ & $222(34.7)$ & $608(32.1)$ & \\
\hline$>20$ & $277(11.0)$ & $64(10.1)$ & $213(11.2)$ & \\
\hline Bilateral & & & & 0.614 \\
\hline Yes & $393(13.7)$ & $103(16.1)$ & $290(15.3)$ & \\
\hline No & $2143(86.3)$ & $536(83.9)$ & $1607(84.7)$ & \\
\hline Tumor location & & & & 0.911 \\
\hline Superior pole & $541(21.3)$ & $135(21.1)$ & $406(21.4)$ & \\
\hline Others & $1995(78.7)$ & $504(78.9)$ & $1491(78.6)$ & \\
\hline Multifocality & & & & 0.812 \\
\hline Presence & $453(17.9)$ & $116(18.2)$ & $337(17.8)$ & \\
\hline Absence & $2083(82.1)$ & $523(81.8)$ & $1560(82.2)$ & \\
\hline Extrathyroid extension & & & & 0.630 \\
\hline Presence & 865 & $223(34.9)$ & $642(33.8)$ & \\
\hline Absence & $1671(65.9)$ & $416(65.1)$ & $1255(66.2)$ & \\
\hline CLT & & & & $<0.001$ \\
\hline Yes & $526(20.7)$ & $68(10.6)$ & $458(24.1)$ & \\
\hline No & $2010(79.3)$ & $571(89.4)$ & $1439(75.9)$ & \\
\hline LNM & & & & $<0.001$ \\
\hline Yes & $1391(54.9)$ & $420(65.7)$ & $971(51.2)$ & \\
\hline No & $1145(45.1)$ & $219(34.3)$ & $926(48.8)$ & \\
\hline CLNM & & & & $<0.001$ \\
\hline Yes & $1341(52.5)$ & $410(64.2)$ & $921(48.6)$ & \\
\hline No & $1205(47.5)$ & $229(35.8)$ & $976(51.4)$ & \\
\hline LLNM & & & & 0.003 \\
\hline Yes & $558(22.0)$ & $168(26.3)$ & $390(20.6)$ & \\
\hline No & $1978(78.0)$ & $471(73.7)$ & $1507(79.4)$ & \\
\hline Recurrence & $29(1.1)$ & $11(1.7)$ & $18(0.9)$ & 0.131 \\
\hline
\end{tabular}

Continuous data are shown as mean \pm standard deviation. CLT, chronic lymphocytic thyroiditis; LNM, lymph node metastasis.

male patients to $40.8 \%$ among female patients in PTMC $(P<0.001)$.

3.3. Subgroup Analysis. We further performed stratified analysis to investigate the associations between gender and CLNM and LLNM among different clinicopathological subgroups. After full adjustment, the stratified analyses indicated that male gender was independently associated with CLNM in all subgroups (Figure 2). However, we observed that there was no significant correlation between gender and LLNM across most subgroups, except for subgroups of patients aged 35-55y $(\mathrm{OR}=1.45,95 \% \mathrm{CI}$ : 1.03-2.03) and patients with a solitary tumor $(\mathrm{OR}=1.34$, 95\% CI: 1.01-1.78; Figure 3).

3.4. Kaplan-Meier Analyses of Recurrence-Free Survival of Patients. The median follow-up time was 30 months (interquartile range $(\mathrm{IQR})=18-43)$. As shown in Figure 4, LNM, age, and tumor size were significantly associated with poorer RFS. However, no differences were found between RFS and genders, ETE, and CLT.

\section{Discussion}

This population-based study in southwestern China elucidated that the prognosis of thyroid cancer is good, and very few patients developed recurrent disease. Males had a higher incidence of LNM, CLNM, and LLNM in PTC than females. Male gender was an independent risk factor of CLNM. After adjustment for confounding factors, the association persisted in all subgroups; whereas, no significant difference of LLNM between female and male patients was found. Additionally, we found male gender was potentially not associated with a worse prognosis.

In this study, univariate analysis showed that there were no differences in terms of age, tumor size, tumor location, and ETE between female patients and males. Nevertheless, the female group had higher percentages of coexistent CLT compared to the male group. This result is consistent with other studies $[25,26]$.

Surgery is one of the most important treatments for PTC. Poor prognosis can be owing to an inadequate extent of lymph node dissection. Thus, the status of lymph nodes is a crucial factor for guiding treatment options, which may direct the surgeon to adjust the actual extent of surgery 
TABLE 2: Univariate and multivariate logistic regression analyses of factors associated with lymph node metastasis.

\begin{tabular}{|c|c|c|c|c|}
\hline \multirow{2}{*}{ Variables } & \multicolumn{2}{|c|}{ Univariate analysis } & \multicolumn{2}{|c|}{ Multivariate analysis } \\
\hline & OR (95\% CI) & $P$ & OR $(95 \% \mathrm{CI})$ & $P$ \\
\hline Gender (male/female) & $1.83(1.52-2.20)$ & $<0.001$ & $1.93(1.59-2.35)$ & $<0.001$ \\
\hline Age $(y)$ & & $<0.001$ & & $<0.001$ \\
\hline$<35$ & Reference & & Reference & \\
\hline $35-55$ & $0.56(0.47-0.68)$ & $<0.001$ & $0.58(0.48-0.70)$ & $<0.001$ \\
\hline$\geq 55$ & $0.46(0.36-0.59)$ & $<0.001$ & $0.42(0.33-0.55)$ & $<0.001$ \\
\hline Tumor size (mm) & & $<0.001$ & & $<0.001$ \\
\hline$\leq 10$ & Reference & & Reference & \\
\hline $10-20$ & $2.24(1.88-2.67)$ & $<0.001$ & $2.09(1.75-2.52)$ & $<0.001$ \\
\hline$>20$ & $4.47(3.30-6.06)$ & $<0.001$ & $4.18(3.06-5.73)$ & $<0.001$ \\
\hline $\operatorname{ETE}(+/-)$ & $1.49(1.27-1.77)$ & $<0.001$ & $1.31(1.10-1.57)$ & 0.003 \\
\hline Multifocality (+/-) & $2.01(1.62-2.49)$ & $<0.001$ & $1.92(1.53-2.41)$ & $<0.001$ \\
\hline \multicolumn{5}{|l|}{ Tumor location } \\
\hline Superior pole & Reference & 0.980 & & \\
\hline Others & $0.99(0.82-1.21)$ & & & \\
\hline Bilateral $(+/-)$ & $2.24(1.77-2.83)$ & $<0.001$ & & \\
\hline $\operatorname{CLT}(+/-)$ & $0.91(0.75-1.11)$ & 0.349 & & \\
\hline
\end{tabular}

CLT, chronic lymphocytic thyroiditis; ETE, extrathyroid extension; -, negative; +, positive.

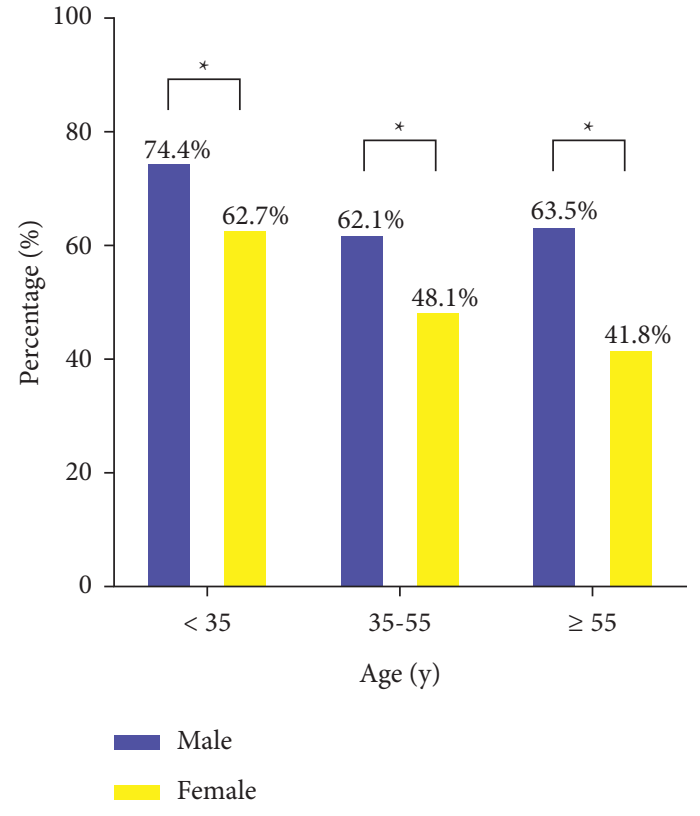

(a)

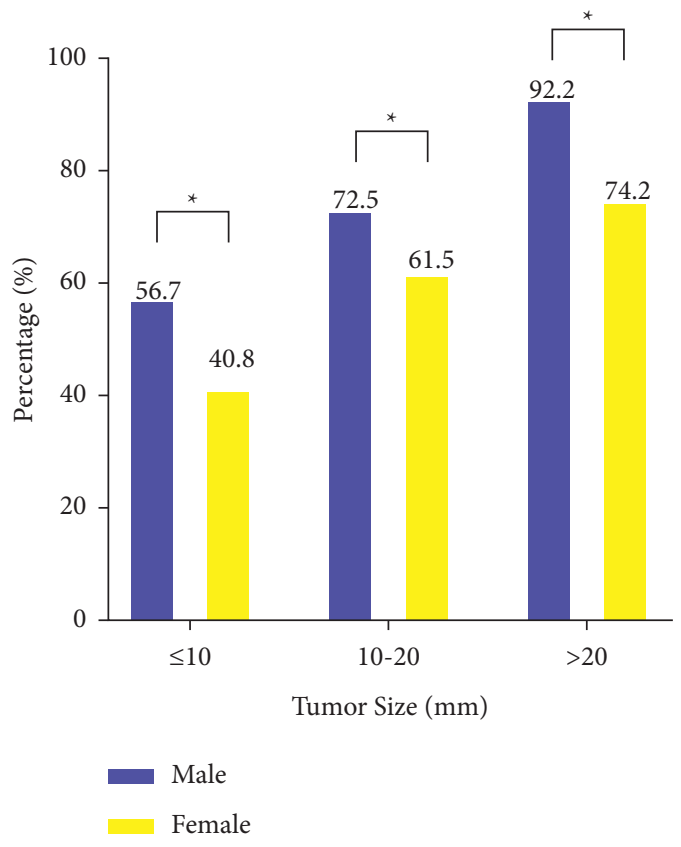

(b)

FIGURE 1: Comparisons of overall lymph node metastasis rates between female and male PTC patients when stratified by (a) age and (b) tumor size.

$[26,27]$. Whether male gender has an influence on LNM of patients with PTC is worthy of attention as, if so, a more aggressive treatment should be adopted. However, aggressive treatment may result in more complications, such as injury of recurrent laryngeal nerves and hypoparathyroidism [9]. It is important to clarify any correlation of gender and LNM. In a research study including 2930 patients with PTC, Lee et al. reported that male gender is a strong independent prognostic factor in patients with PTC, but not in PTMC patients [9]. Nevertheless, our observational study showed that male gender was a strong independent risk factor of LNM not only for PTC but also for PTMC. We further divided the patients into three groups based on tumor size and age to reduce the effect of those two clinicopathological parameters on the correlation between LNM and gender. We observed that the overall rate of lymph node metastasis rose with increasing tumor size, while older age was a protective factor for LNM. Furthermore, the LNM rate in male patients was always higher than in female patients (all $P<0.001$ ). In summary, our findings demonstrated that 


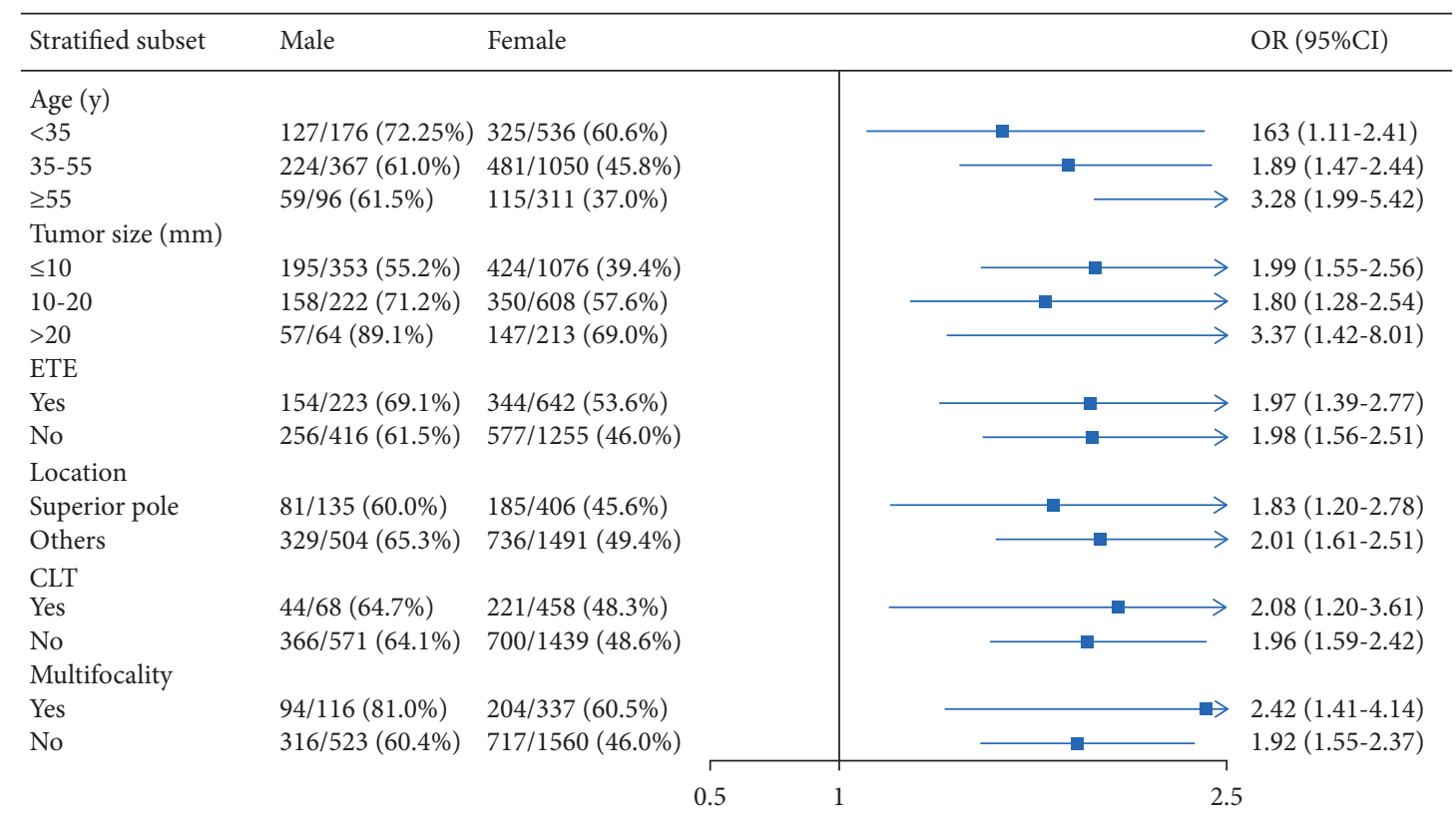

FIgure 2: The forest plot for OR comparing central lymph node metastasis between female and male PTC patients according to different variables. ETE, extrathyroid extension; CLT, chronic lymphocytic thyroiditis; OR, odds ratio; CI, confidence interval.

\begin{tabular}{|c|c|c|c|c|c|}
\hline \multirow{2}{*}{$\begin{array}{l}\text { Stratified subset } \\
\text { Age (y) }\end{array}$} & \multirow[t]{2}{*}{ Male } & \multicolumn{3}{|l|}{ Female } & \multirow[t]{2}{*}{ OR $(95 \% \mathrm{CI})$} \\
\hline & & & & & \\
\hline$<35$ & $59 / 176(33.5 \%)$ & $152 / 536(28.4 \%)$ & $\longrightarrow$ & & $0.95(0.60-1.49)$ \\
\hline $35-55$ & $90 / 367(24.5 \%)$ & $177 / 1050(16.9 \%)$ & & $\longrightarrow$ & $1.45(1.03-2.03)$ \\
\hline$\geq 55$ & $19 / 96(19.8 \%)$ & $61 / 311(19.6 \%)$ & $\longleftrightarrow$ & & $0.70(0.35-1.42)$ \\
\hline \multicolumn{6}{|l|}{ Tumor size (mm) } \\
\hline$\leq 10$ & $51 / 353(14.4 \%)$ & $114 / 1076(10.6 \%)$ & & $\longrightarrow$ & $1.20(0.81-1.78)$ \\
\hline $10-20$ & $79 / 222(35.6 \%)$ & $170 / 608(28.0 \%)$ & & $\rightarrow$ & $1.12(0.76-1.63)$ \\
\hline$>20$ & $38 / 64(59.4 \%)$ & $106 / 213(49.8 \%)$ & & & $1.02(0.54-1.95)$ \\
\hline \multicolumn{6}{|c|}{ 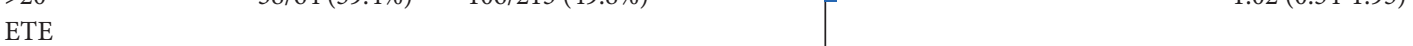 } \\
\hline Yes & $85 / 223(38.1 \%)$ & $198 / 642(30.8 \%)$ & & $\longrightarrow$ & $1.18(0.80-1.75)$ \\
\hline No & $83 / 416(20.0 \%)$ & $192 / 1255(15.3 \%)$ & & $\longrightarrow$ & $1.14(0.82-1.58)$ \\
\hline \multicolumn{6}{|l|}{ Location } \\
\hline Superior pole & 50/135 (37.0\%) & $127 / 406(31.3 \%)$ & & $\rightarrow-$ & $1.10(0.67-1.82)$ \\
\hline Others & $118 / / 504(23.4 \%)$ & $263 / 1491(17.6 \%)$ & & $\longrightarrow$ & $1.16(0.87-1.55)$ \\
\hline \multicolumn{6}{|c|}{ 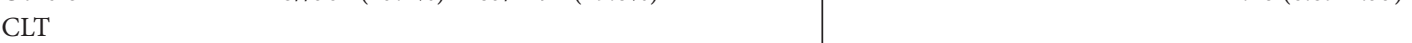 } \\
\hline Yes & $20 / 68(29.4 \%)$ & $104 / 458(22.7 \%)$ & & $\rightarrow$ & $1.07(0.54-2.15)$ \\
\hline No & $148 / 571(25.9 \%)$ & $286 / 1439(19.9 \%)$ & & $\longrightarrow$ & $1.16(0.88-1.52)$ \\
\hline \multicolumn{6}{|l|}{ Multifocality } \\
\hline Yes & $40 / 116(34.5 \%)$ & $114 / 337(33.8 \%)$ & $\longleftrightarrow$ & - & $0.67(0.39-1.14)$ \\
\hline No & $128 / 523(24.5 \%)$ & $276 / 1560(17.7 \%)$ & & 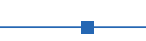 & $1.34(1.01-1.78)$ \\
\hline \multicolumn{6}{|l|}{ CLNM } \\
\hline Yes & $158 / 410(38.5 \%)$ & $340 / 921(36.9 \%)$ & & $\longrightarrow$ & $1.01(0.48-2.10)$ \\
\hline \multirow[t]{2}{*}{ No } & $10 / 229(4.4 \%)$ & $50 / 976(5.1 \%)$ & $\longleftarrow$ & & $1.17(0.90-1.53)$ \\
\hline & & & 0.5 & 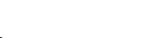 & 2.5 \\
\hline
\end{tabular}

FIGURE 3: The forest plot for OR comparing lateral lymph node metastasis between female and male PTC patients according to different variables. ETE, extrathyroid extension; CLT, chronic lymphocytic thyroiditis; CLNM, central lymph node metastasis; OR, odds ratio; CI, confidence interval.

LNM occurred more frequently in young female or male patients with larger tumors. Furthermore, gender may have implications for the surgical management of PTC.
Many studies address the rate and risk factors of CLNM $[17,27,28]$. Sporadic studies indicated an association of male gender with CLNM [24]; however, little attention specifically 


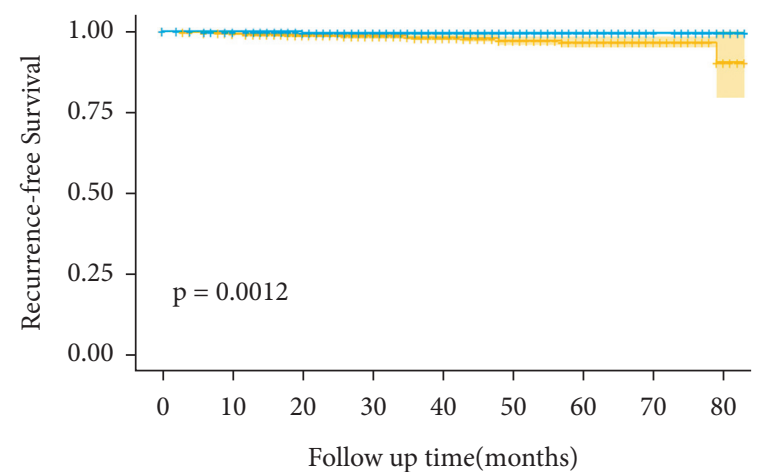

Number at risk

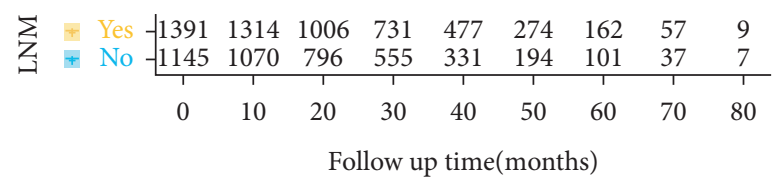

(a)
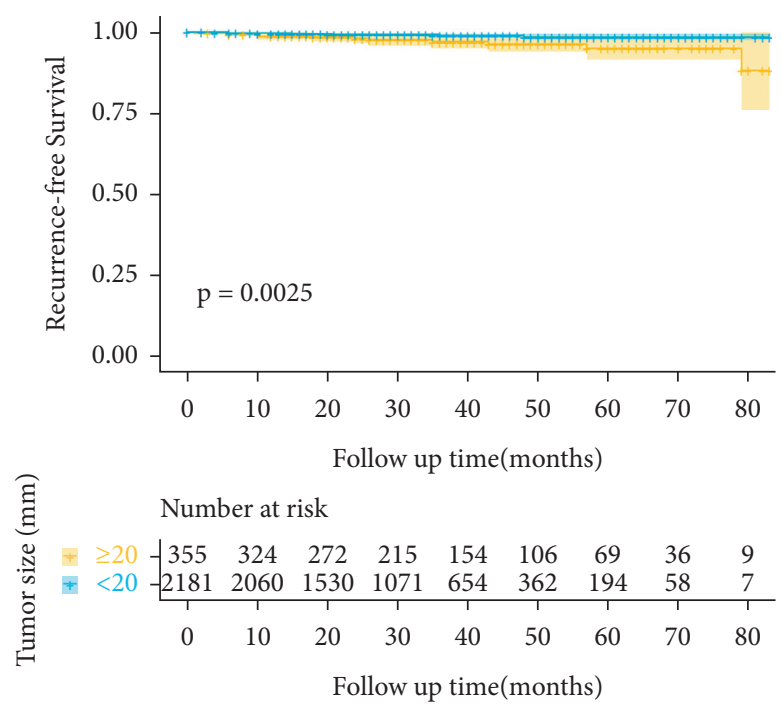

(c)

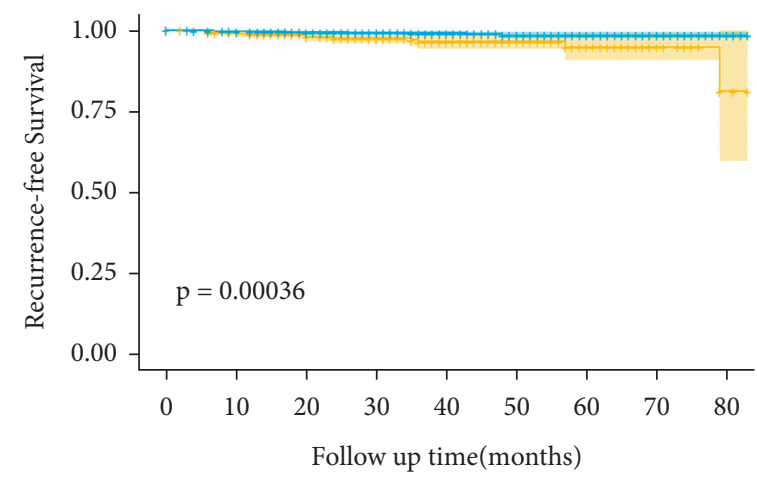

Number at risk

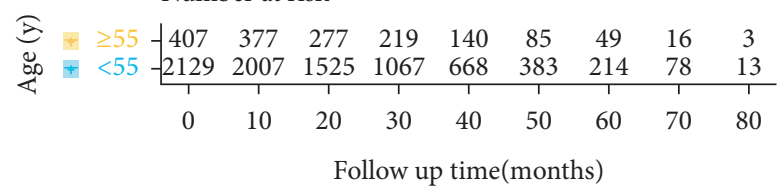

(e)

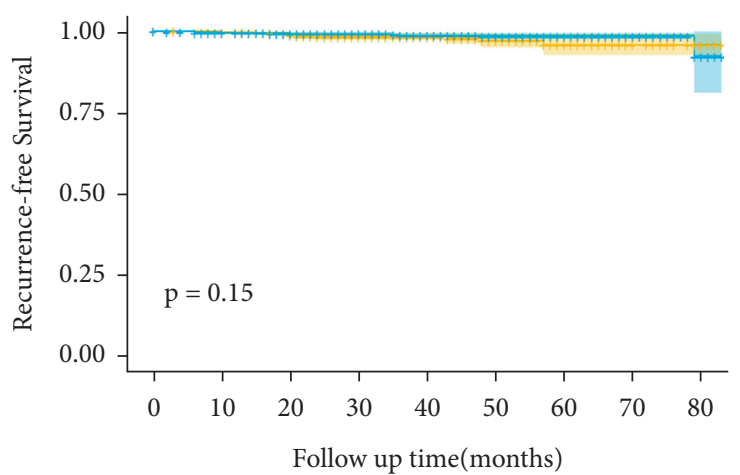

Number at risk

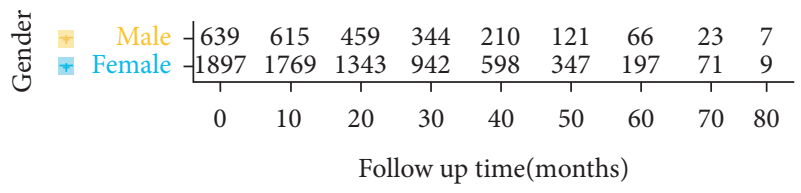

(b)

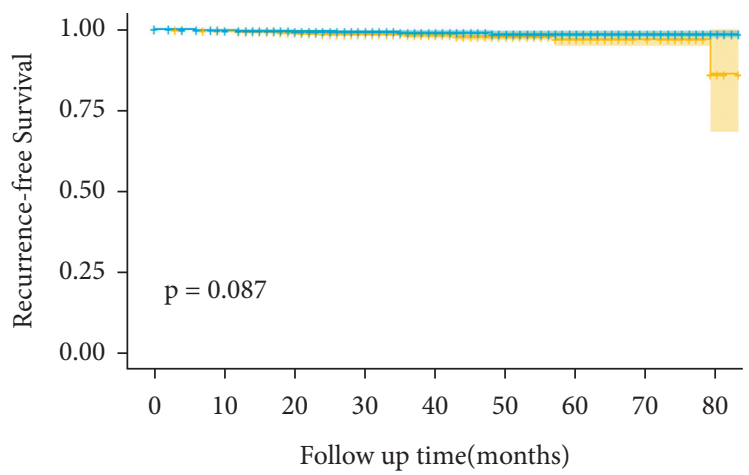

Number at risk

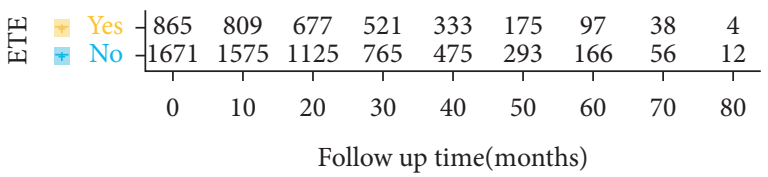

(d)

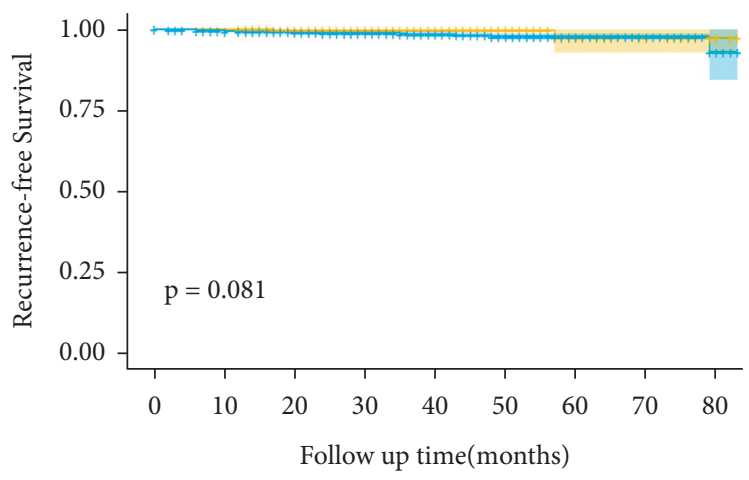

Number at risk

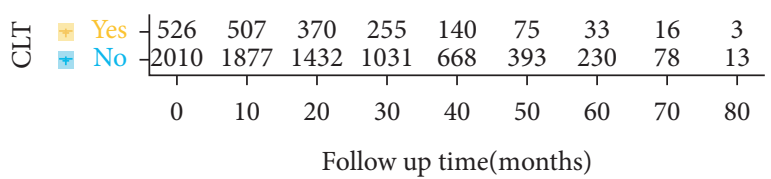

(f)

Figure 4: The Kaplan-Meier curves of recurrence-free survival according to (a) lymph node status, (b) gender, (c) tumor size, (d) extrathyroid extension, (e) age, and (f) chronic lymphocytic thyroiditis. LNM, lymph node metastasis. ETE, extrathyroid extension; CLT, chronic lymphocytic thyroiditis. 
focuses on this relationship. We conducted a stratified analysis and found that male gender was a stable risk factor for CLNM in all subgroups. In view of this result, we suggest that central lymph node dissection for male patients should be thoroughly and carefully evaluated by the surgeon during surgery. Male gender is considered positively associated with LLNM in some studies $[11,29]$, while such a relationship is not observed in many other reports $[30,31]$. We also investigated the correlation between gender and LLNM. Univariate analysis found that male patients had a higher incidence of LLNM. However, no association was found between gender and LLNM in subgroup analysis after adjustment for other clinicopathological factors. Therefore, a gender factor should not be used to determine the strategy of lateral lymph node dissection.

Many research studies report that LNM is an independent prognostic factor for PTC $[21,32,33]$. Consistent with those studies, we confirmed that LNM was associated with poor prognosis. Male gender is generally thought to be closely related to recurrence of PTC $[9,11,33,34]$. Ito et al. reported that male gender is a risk factor for lymph node recurrence [35]. Meanwhile, a meta-analysis, including 13 articles, reports that male gender is a poor independent prognostic factor in PTC patients [10]. However, some studies found that male gender is not associated with recurrence-free survival [13, 15, 36, 37]. The Kaplan-Meier analyses of our data indicated that male gender was not correlated with RFS. Based on this, we currently cannot ascertain that gender is or is not an independent survival risk factor in thyroid cancer and that male gender should be taken into consideration as a prognostic factor for risk classification. Certainly, the relatively short follow-up time could have influenced this result; hence, long-term followup and Cox proportional hazards regression for adjustment for a large number of confounders is needed to clarify the results. Moreover, future studies are warranted to elucidate the sex-specific molecular mechanisms regarding the role of gender played in tumorigenesis and development of PTC which may assist clinicians with therapeutic decisions $[4,38]$.

Therefore, while the association of prognosis with gender is controversial, our data provide important evidence to support this issue. The current study utilizes a large sample size to investigate the influence of gender on LNM and risk factors affecting the locoregional recurrence of PTC patients from southwestern China. Furthermore, we have adequately adjusted for confounding factors in multivariable analysis assessing the association between gender and LNM to generate robust results.

The limitations of the study are as follows. First, this was a retrospective study rather than a prospective cohort, so the quality of evidence was limited. Second, due to the short follow-up duration and a considerable portion of early stage PTC, the recurrence outcome was rare. Because of this, we could not perform Cox proportional hazards regression to assess the independent effects of gender on prognosis. A reasonable follow-up is needed to evaluate recurrence. Additionally, this was an observational study of a single center, specifically for a population in southwestern China, which should not be extrapolated to other regions. Hence, it is necessary to use real-world, multicenter data covering a larger number of patients to verify our findings.

\section{Conclusions}

In this observational cohort study of patients with PTC from southwestern China, male gender was independently associated with CLNM but potentially not with LLNM after sufficient adjustment for confounders. Moreover, currently, there is no sufficient evidence to conclude that gender was associated with recurrence. Further long-term follow-up data in regards to recurrence/death are required to assist with risk stratification.

\section{Abbreviations}

PTC: Papillary thyroid carcinoma

PTMC: Papillary thyroid microcarcinoma

LNM: Lymph node metastasis

CLNM: Central lymph node metastasis

LLNM: Lateral lymph node metastasis

ETE: Extrathyroid extension

CLT: Chronic lymphocytic thyroiditis

RFS: Recurrence-free survival

OR: Odds ratio

CI: Confidence interval.

\section{Data Availability}

The datasets used and analyzed in the present study are available from the corresponding author upon request.

\section{Ethical Approval}

The study was in accordance with the 1964 Helsinki declaration and approved by the Institutional Review Board of First Affiliated Hospital of Chongqing Medical University.

\section{Consent}

Written informed consent was signed by all of the patients.

\section{Disclosure}

Jiang Zhu and Rui Huang are the co-first authors.

\section{Conflicts of Interest}

The authors declare that they have no conflicts of interest.

\section{Authors' Contributions}

JZ and XS conceived and designed the study. JZ, RH, and HR analyzed the patient data. JZ and RH wrote and revised the article. XS, JZ, and RH conceived and designed the study. JZ, $\mathrm{RH}, \mathrm{PY}$, and HR analyzed the patient data. JZ and RH wrote and revised the manuscript and contributed equally to this work. All authors read and approved the manuscript. 


\section{Acknowledgments}

This work was supported by grants from Chongqing Science and Technology Committee (cstc2017shmsA1035).

\section{References}

[1] L. Davies and H. G. Welch, "Current thyroid cancer trends in the United States," JAMA Otolaryngology-Head \& Neck Surgery, vol. 140, no. 4, pp. 317-322, 2014.

[2] Y. Deng, H. Li, M. Wang et al., "Global burden of thyroid cancer from 1990 to 2017," JAMA Network Open, vol. 3, no. 6, Article ID e208759, 2020.

[3] M. H. Hajeer, H. A. Awad, N. I. Abdullah, G. H. Almuhaisen, and L. E. Abudalu, "The rising trend in papillary thyroid carcinoma," Saudi Medical Journal, vol. 39, no. 2, pp. 147-153, 2018.

[4] C. M. Lopes-Ramos, J. Quackenbush, and D. L. DeMeo, "Genome-wide sex and gender differences in cancer," Frontiers in Oncology, vol. 10, 2020.

[5] W. Chen, R. Zheng, T. Zuo, H. Zeng, S. Zhang, and J. He, "National cancer incidence and mortality in China, 2012," Chinese Journal of Cancer Research, vol. 28, no. 1, pp. 1-11, 2016.

[6] R. L. Siegel, K. D. Miller, H. E. Fuchs, and A. Jemal, "Cancer statistics, 2021," CA: A Cancer Journal for Clinicians, vol. 71, no. 1, pp. 7-33, 2021.

[7] M. Huang, C. Yan, H. Wei, Y. Lv, and R. Ling, "Clinicopathological characteristics and prognosis of thyroid cancer in northwest China: a population-based retrospective study of 2490 patients," Thoracic Cancer, vol. 9, no. 11, pp. 1453-1460, 2018.

[8] B. A. Kilfoy, S. S. Devesa, M. H. Ward et al., "Gender is an agespecific effect modifier for papillary cancers of the thyroid gland," Cancer Epidemiology Biomarkers and Prevention, vol. 18, no. 4, pp. 1092-1100, 2009.

[9] Y. H. Lee, Y. M. Lee, T. Y. Sung et al., "Is male gender a prognostic factor for papillary thyroid microcarcinoma?" Annals of Surgical Oncology, vol. 24, no. 7, pp. 1958-1964, 2017.

[10] K. Guo and Z. Wang, "Risk factors influencing the recurrence of papillary thyroid carcinoma: a systematic review and metaanalysis," International Journal of Clinical and Experimental Pathology, vol. 7, no. 9, pp. 5393-5403, 2014.

[11] J. Ding, W. Wu, J. Fang, J. Zhao, and L. Jiang, "Male sex is associated with aggressive behaviour and poor prognosis in Chinese papillary thyroid carcinoma," Scientific Reports, vol. 10, no. 1, Article ID 4141, 2020.

[12] A. Zahedi, L. Bondaz, M. Rajaraman et al., "Risk for thyroid cancer recurrence is higher in men than in women independent of disease stage at presentation," Thyroid, vol. 30, no. 6, pp. 871-877, 2020.

[13] E. Yorke, A. Melck, and S. M. Wiseman, "Impact of sex on the clinicopathological characteristics and prognosis of papillary thyroid cancer," Canadian Journal of Surgery, vol. 59, no. 4, pp. 287-288, 2016.

[14] N. Nilubol, L. Zhang, and E. Kebebew, "Multivariate analysis of the relationship between male sex, disease-specific survival, and features of tumor aggressiveness in thyroid cancer of follicular cell origin," Thyroid, vol. 23, no. 6, pp. 695-702, 2013.

[15] S. L. Oyer, V. A. Smith, and E. J. Lentsch, "Sex is not an independent risk factor for survival in differentiated thyroid cancer," The Laryngoscope, vol. 123, no. 11, pp. 2913-2919, 2013.

[16] Y. Huang, S. Qu, G. Zhu et al., "BRAF V600E mutationassisted risk stratification of solitary intrathyroidal papillary thyroid cancer for precision treatment," Journal of the $\mathrm{Na}$ tional Cancer Institute: Journal of the National Cancer Institute, vol. 110, no. 4, pp. 362-370, 2018.

[17] J.-D. Shou, F.-B. Li, L.-H. Shi, L. Zhou, L. Xie, and J.-B. Wang, "Predicting non-small-volume central lymph node metastases ( $>5$ or $\geq 2 \mathrm{~mm}$ ) preoperatively in $\mathrm{cN0}$ papillary thyroid microcarcinoma without extrathyroidal extension," Medicine, vol. 99, no. 38, Article ID e22338, 2020.

[18] S. Lu, R. Zhao, Y. Ni et al., "Development and validation of a nomogram for preoperative prediction of cervical lymph node involvement in thyroid microcarcinoma," Aging, vol. 12, no. 6, pp. 4896-4906, 2020.

[19] Y. Heng, Z. Yang, L. Zhou, J. Lin, W. Cai, and L. Tao, "Risk stratification for lateral involvement in papillary thyroid carcinoma patients with central lymph node metastasis," Endocrine, vol. 68, no. 2, pp. 320-328, 2020.

[20] G. Mercante, A. Frasoldati, C. Pedroni et al., "Prognostic factors affecting neck lymph node recurrence and distant metastasis in papillary microcarcinoma of the thyroid: results of a study in 445 patients," Thyroid, vol. 19, no. 7, pp. 707-716, 2009.

[21] Y. J. Suh, H. Kwon, S.-J. Kim et al., "Factors affecting the locoregional recurrence of conventional papillary thyroid carcinoma after surgery: a retrospective analysis of 3381 patients," Annals of Surgical Oncology, vol. 22, 2015.

[22] J. Zhu, R. Huang, D. Hu et al., "Individualized prediction of metastatic involvement of lymph nodes posterior to the right recurrent laryngeal nerve in papillary thyroid carcinoma," OncoTargets and Therapy, vol. 12, pp. 9077-9084, 2019.

[23] J. Zhu, R. Huang, P. Yu et al., "Clinical implications of Delphian lymph node metastasis in papillary thyroid carcinoma," Gland Surgery, vol. 10, no. 1, pp. 73-82, 2021.

[24] J. Zhu, J. Zheng, L. Li et al., "Application of machine learning algorithms to predict central lymph node metastasis in T1-T2, non-invasive, and clinically node negative papillary thyroid carcinoma," Frontiers of Medicine, vol. 8, Article ID 635771, 2021.

[25] X. Wen, B. Wang, Q. Jin, W. Zhang, and M. Qiu, “Thyroid Antibody status is associated with central lymph node metastases in papillary thyroid carcinoma patients with hashimoto's thyroiditis," Annals of Surgical Oncology, vol. 26, no. 6, pp. 1751-1758, 2019.

[26] E. Song, M. J. Jeon, S. Park et al., "Influence of coexistent Hashimoto's thyroiditis on the extent of cervical lymph node dissection and prognosis in papillary thyroid carcinoma," Clinical Endocrinology, vol. 88, no. 1, pp. 123-128, 2018.

[27] W. Sun, X. Lan, H. Zhang et al., "Risk factors for central lymph node metastasis in CNO papillary thyroid carcinoma: a systematic review and meta-analysis," PLoS One, vol. 10, no. 10, Article ID e0139021, 2015.

[28] B. Ma, Y. Wang, S. Yang, and Q. Ji, "Predictive factors for central lymph node metastasis in patients with cN0 papillary thyroid carcinoma: a systematic review and meta-analysis," International Journal of Surgery, vol. 28, pp. 153-161, 2016.

[29] S. Zhan, D. Luo, W. Ge, B. Zhang, and T. Wang, "Clinicopathological predictors of occult lateral neck lymph node metastasis in papillary thyroid cancer: a meta-analysis," Head \& Neck, vol. 41, no. 7, pp. 2441-2449, 2019.

[30] M. Song, Z. Huang, S. Wang et al., "Predictive factors of lateral lymph node metastasis in conventional papillary thyroid carcinoma," Gland Surgery, vol. 9, no. 4, pp. 1000-1007, 2020. 
[31] K. Back, J. S. Kim, J.-H. Kim, and J.-H. Choe, "Superior located papillary thyroid microcarcinoma is a risk factor for lateral lymph node metastasis," Annals of Surgical Oncology, vol. 26, no. 12, pp. 3992-4001, 2019.

[32] F. Bian, C. Li, D. Han, F. Xu, and J. Lyu, "Competing-risks model for predicting the postoperative prognosis of patients with papillary thyroid adenocarcinoma based on the surveillance, epidemiology, and end results (SEER) database," Medical Science Monitor, vol. 26, Article ID e924045, 2020.

[33] Z. Y. Rui, Y. Liu, W. Zheng et al., "A retrospective study of the risk factors and the prognosis in patients with papillary thyroid carcinoma depending on the number of lymph node metastasis," Clinical and Experimental Medicine, vol. 21, 2021.

[34] A. A. Póvoa, E. Teixeira, M. R. Bella-Cueto et al., "Clinicopathological features as prognostic predictors of poor outcome in papillary thyroid carcinoma," Cancers, vol. 12, no. 11, Article ID 3186, 2020.

[35] Y. Ito, T. Higashiyama, Y. Takamura et al., "Risk factors for recurrence to the lymph node in papillary thyroid carcinoma patients without preoperatively detectable lateral node metastasis: validity of prophylactic modified radical neck dissection," World Journal of Surgery, vol. 31, no. 11, pp. 2085-2091, 2007.

[36] J.-W. Feng, X.-H. Yang, B.-Q. Wu, D.-L. Sun, Y. Jiang, and $\mathrm{Z}$. $\mathrm{Qu}$, "Predictive factors for central lymph node and lateral cervical lymph node metastases in papillary thyroid carcinoma," Clinical and Translational Oncology, vol. 21, no. 11, pp. 1482-1491, 2019.

[37] M. S. Papageorgiou, N. Liratzopoulos, M. Karanikas, E. I. Efremidou, G. Minopoulos, and K. J. Manolas, "Epidemiology and survival of patients with well-differentiated thyroid cancer subjected to total thyroidectomy in Thrace area, Northern Greece: a 20 year experience," Minerva Chirurgica, vol. 65, no. 2, pp. 137-143, 2010.

[38] R. Han, W. Sun, J. Huang, L. Shao, and H. Zhang, "Sex-biased DNA methylation in papillary thyroid cancer," Biomarkers in Medicine, vol. 15, no. 2, pp. 109-121, 2021. 